\title{
Funciones profesionales de los trabajadores sociales en España
}

\author{
Professional functions of social workers in Spain
}

\author{
Alberto BALLESTERO IZQUIERDO \\ Juan Jesús ViscARret GARRO \\ María Jesús ÚRIZ PEMÁN \\ Grupo EFIMEC \\ Universidad Pública de Navarra \\ efimec@unavarra.es
}

Recibido: 12/03/2012

Revisado: 07/09/2012

Aceptado: 02/01/2013

Disponible on line: 16/04/2013

\section{Resumen}

Los resultados presentados en este artículo forman parte de un estudio de ámbito nacional que, por primera vez en España, ha encuestado a las profesionales del Trabajo Social colegiadas para conocer su práctica profesional cotidiana, según la clasificación de funciones ofrecida en el Libro Blanco de Trabajo Social ${ }^{1}$. Los resultados se ofrecen en tres partes: una descriptiva de las tendencias generales; la segunda, un análisis factorial para un agrupamiento y clasificación tipológicos de las funciones de las trabajadoras sociales; y la tercera, un análisis de conglomerados para agrupar a las trabajadoras sociales según sus funciones en torno a cuatro perfiles profesionales mayoritarios: gestión-planificación, intervención para el cambio, intervención asistencial y académico. Los resultados apuntan a la necesidad de reflexionar sobre el futuro de la actividad de las trabajadoras sociales en la sociedad y sobre la orientación académica del Grado en Trabajo Social, ya que la formación universitaria puede atender a las necesidades de un «mercado laboral» de lo social, con funciones de marcado carácter administrativo y de gestión, o puede formar para el conjunto de funciones que la tradición reflexiva de la disciplina (investigación) y la evolución histórica de la profesión han configurado como esenciales para la misma (intervención).

Palabras clave: funciones, profesión, Trabajo Social, trabajadoras sociales, España.

\begin{abstract}
The results presented here are a part of a nationwide study which has surveyed, for the first time in Spain, female social workers registered in their official Associations in order to identify their daily practice, according to the classification of functions contained in the White Paper of Social Work. The results are presented in three sections: a) a description of general trends, b) a factor analysis for a typological grouping and classification of the functions of social workers and c) a cluster analysis aimed to group the social workers according to their functions into four main professional profiles, namely: management-planning, intervention for change, care intervention and academic. The results of the research point to the need to reflect on the future of the activity of female social workers in society and the academic orientation of the Degree in Social Work, since university education can meet the needs of a «labor market in the social field», with mainly an administrative and management character, or it may train the professionals to perform all of the functions that the reflective tradition of the discipline (research) and the historical evolution of the profession have set as essential to it (intervention).
\end{abstract}

Keywords: functions; profession; social work; social workers; Spain.

Referencia personalizada: Ballestero Izquierdo, A., Viscarret Garro, J. J., y Úriz Pemán, M. J. (2013): «Funciones profesionales de los trabajadores sociales en España». Cuadernos de Trabajo Social, 26(1): 127-138.

Sumario: Introducción. 1. Método. 2. Resultados. 3. Discusión y conclusiones. 4. Referencias bibliográficas.

${ }^{1}$ Trabajo financiado por el proyecto FFI2008-05546, Dilemas éticos en la intervención social. La perspectiva de los trabajadores sociales en España, del Ministerio de Ciencia e Innovación del Gobierno de España. 


\section{Introducción}

Todas las profesiones intentan redefinir y asegurar unas funciones y unos conocimientos especializados como fundamento de su realización profesional. El concepto de función surge de la naturaleza misma del proceso social del trabajo, permitiendo identificar la aportación que hace a la sociedad una profesión, describiendo su finalidad y estableciendo sus límites. Todas las profesiones encuentran su razón de ser en las necesidades sociales y en toda colectividad existe la división de funciones entre personas o grupos, de modo que cada cual realice una contribución específica al conjunto de la sociedad. Portuondo (1983) define la función como «el tipo de actividad laboral que caracteriza e identifica el trabajo socialmente útil que realiza el hombre para alcanzar un objetivo determinado». Desde esta perspectiva, la función comprende la esfera de responsabilidad, que establecen las actividades relacionadas entre sí y encaminadas a un fin, y que determina el ejercicio de una profesión. Estas deben reflejar el conjunto de problemas que las profesionales deben ser capaces de enfrentar, analizar y resolver. Ante estas reflexiones, se asume el concepto de función como el papel desempeñado, reconocido y aceptado por la sociedad para los miembros de una disciplina, que se materializa en las actividades o tareas que determinan el ejercicio de una profesión (Torres Esperón, 2008).

La identidad profesional de las trabajadoras sociales se arraiga en el pasado, donde construye las primeras identificaciones que dan origen a la profesión y que les permiten desempeñar un papel en la sociedad. Producto de este proceso dialéctico, la profesión ha ido enriqueciendo y modificando su perfil profesional en la medida en que ha incorporado las aportaciones procedentes de los nuevos fenómenos y demandas sociales a los que ha tenido que ir haciendo frente, teniendo acceso a nuevas funciones y desempeñando nuevos roles, aumentando su caudal de conocimientos y profundizando la reflexión sobre la propia práctica profesional.

En España se constata que, producto de la explosión de nuevas necesidades sociales surgidas como consecuencia del proceso de modernización del país, los trabajadores sociales han ido incrementado su presencia en nuevos y diferentes ámbitos profesionales, que les exigen nuevas competencias. Ello ha dificultado la definición de las funciones que desempeñan las trabajadores sociales en sus puestos de trabajo. Para tal fin, y con motivo de la adaptación de los estudios de Trabajo Social al Espacio Europeo de Educación Superior, se realizó en España un estudio pormenorizado de la legislación europea, nacional y autonómica, por ser donde se regulan y quedan normadas las funciones que deben desempeñar las profesionales del Trabajo Social. Dicho estudio y análisis, que fue llevado a cabo por profesionales del Trabajo Social, del Colegio Nacional de Trabajadores Sociales, y por personas del ámbito universitario, cristalizó en la realización del Libro Blanco de Trabajo Social (Vázquez, 2004) donde, entre otros aspectos, se realiza un análisis del perfil profesional de las trabajadoras sociales. Allí se definen las funciones y competencias de los trabajadores sociales a través de las cuales se estructura la formación del Trabajo Social en España y se adapta al Espacio Europeo de Educación Superior. Realizado el análisis desde los marcos normativos propios de la profesión y de la disciplina científica se constata que las funciones profesionales de los trabajadores sociales en España son las siguientes:

1. Función preventiva. Actuación precoz sobre las causas que generan problemáticas individuales y colectivas. Elaboración y ejecución de proyectos de intervención para grupos de población en situaciones de riesgo social y de carencia de aplicación de los derechos humanos.

2. Función de atención directa. Responde a la atención de individuos o grupos que presentan o están en riesgo de presentar, problemas de índole social. Su objeto será potenciar el desarrollo de las capacidades y facultades de las personas, para afrontar por sí mismas futuros problemas e integrarse satisfactoriamente en la vida social.

3. Función de planificación. Es la acción de ordenar y conducir un plan de acuerdo con unos objetivos propuestos, contenidos en un programa determinado mediante un proceso de análisis de la realidad y del cálculo de las probables evoluciones de la misma. Esta función se puede desarrollar a dos niveles: microsocial, 
que comprende el diseño de tratamientos, intervenciones y proyectos sociales, y macrosocial, que comprende el diseño de programas y servicios sociales.

4. Función docente. Con el objetivo de impartir enseñanzas teóricas y prácticas de Trabajo Social y de Servicios Sociales, tanto en las propias escuelas universitarias de Trabajo Social, como en otros ámbitos académicos, así como contribuir a la formación teóricopráctica de grado y posgrado de estudiantes de Trabajo Social y de otras disciplinas afines. Las tituladas en Trabajo Social o Asistente Social son las profesionales idóneas para impartir la docencia en las materias de Trabajo Social y Servicios Sociales.

\section{Función de promoción e inserción so-} cial. Se realiza mediante actuaciones encaminadas a restablecer, conservar y mejorar las capacidades, la facultad de autodeterminación y el funcionamiento individual o colectivo. También mediante el diseño y la implementación de las políticas sociales que favorezcan la creación y reajuste de servicios y recursos adecuados a la cobertura de las necesidades sociales.

6. Función de supervisión. Proceso dinámico de capacitación mediante el cual las tituladas en Trabajo Social o Asistente Social responsables de la ejecución de una parte del programa de un servicio, reciben la ayuda de una profesional del Trabajo Social con la finalidad de aprovechar de la mejor forma posible sus conocimientos y habilidades y de perfeccionar sus aptitudes de forma que ejecuten sus tareas profesionales de un modo más eficiente y con mayor satisfacción, tanto para ellas mismas como para el servicio.

7. Función de evaluación. Tiene la finalidad de constatar los resultados obtenidos en las distintas actuaciones, en relación con los objetivos propuestos, teniendo en cuenta técnicas, medios y tiempo empleados.

8. Función gerencial. Se desarrolla cuando la trabajadora social tiene responsabilidades en la planificación de centros, organización, dirección y control de programas sociales y servicios sociales.

9. Función de investigación. Proceso metodológico que lleva a descubrir, describir, interpretar, explicar y valorar una realidad, a través de un trabajo sistematizado de recogida de datos, establecimiento de hipótesis y verifica- ción de las mismas, empleando para ello técnicas profesionales y científicas con el fin de contextualizar una adecuada intervención $\mathrm{y} / \mathrm{o}$ acción social planificada.

10. Función de coordinación. Para determinar mediante la metodología adecuada las actuaciones de un grupo de profesionales, dentro de una misma organización o pertenecientes a diferentes organizaciones, a través de la concertación de medios, técnicas y recursos, a fin de determinar una línea de intervención social y objetivos comunes con relación a un grupo poblacional, comunidad o caso concreto.

Partiendo de la concreción de dichas funciones, en el presente artículo, vamos a analizar con datos como se concretan las mismas en el colectivo profesional del Trabajo Social en España, compuesto por un 91 por ciento de mujeres y un 9 por ciento de hombres.

\section{Método}

Los resultados presentados en este artículo son parte de un estudio más amplio de ámbito nacional en el marco del proyecto Dilemas éticos en la intervención social (La perspectiva de los trabajadores sociales en España) (FFI200805546), dentro del Programa Nacional de Proyectos de Investigación Fundamental del VI Plan Nacional de $\mathrm{I}+\mathrm{D}+\mathrm{i}$. Por primera vez en España, se ha realizado una encuesta representativa de las profesionales del Trabajo Social colegiadas con el fin de conocer diversos elementos que enmarcan su práctica profesional cotidiana. Anteriormente se realizó un estudio en el ámbito de la Comunidad Foral de Navarra con el mismo cuestionario (Úriz, Ballestero y Urien, 2007) y recientemente otro que también trata el asunto en la Comunidad Autónoma de Castilla y León (Gómez, 2010, pp. 219-220). En la encuesta se dedica uno de los apartados al conocimiento de las funciones que desempeñan los trabajadores sociales en su práctica profesional, acorde con la clasificación de funciones ofrecida en el Libro Blanco de Trabajo Social, ya que se trata de una clasificación sólida fruto de un trabajo científico, consensuada a nivel nacional por profesionales y académicas del Trabajo Social.

A los encuestados se les solicita que den su valoración, en una escala ordinal del 1 a 4 (nada-poco-algo-bastante), a las siguientes fun- 
ciones: 1. Prevención (elaboración y ejecución de proyectos de intervención); 2. Atención directa a individuos; 3 . Atención directa a grupos; 4. Planificación (diseño de proyectos de intervención y programas); 5. Docencia: profesora de Trabajo Social; 6. Docencia: tutora de prácticas; 7. Docencia: impartición de cursos relacionados con el Trabajo Social; 8. Promoción e inserción social 9. Diseño e implantación de políticas sociales para crear o reajustar servicios. 10. Supervisión; 11. Planificación de centros, organización y control de programas o servicios; 12. Diagnóstico social (investigación para la intervención); 13. Evaluación de la intervención constatando los resultados obtenidos; 14. Investigación científica (creación de conocimiento teórico y metodológico); 15. Coordinación (grupos, medios, servicios, recursos); 16. Administrativo-burocrática, no relacionada directamente con el trabajo de intervención profesional (papeleo, teléfono, informes).

- Muestra. La unidad muestral es aleatoria y nominativa. Se obtiene a partir del banco de datos que posee el Consejo General de Colegios Oficiales de Diplomados en Trabajo Social y Asistentes Sociales de España. Aplicando la formula del error muestral para poblaciones finitas, significa que para un nivel de confianza de dos sigmas (95,5 por ciento), en donde $\mathrm{p}=\mathrm{q}=0,50$ donde el universo de referencia es de 8.505 profesionales y la muestra que se obtiene $n=700$, el error muestral que se obtiene es de $\pm 3,5$ por ciento. El trabajo de campo se llevó a cabo durante el último trimestre de 2009.

- Análisis. El análisis de los datos ha sido realizado con la aplicación PASW Statistics. Para la consecución de los objetivos planteados, se ha procedido a la realización de un análisis factorial y un análisis de conglomerados (Cluster Analysis) que ha permitido detectar la existencia de grupos o conglomerados, lo más homogéneos en sí y lo más heterogéneos entre sí con respecto a la distribución de las variables analizadas (Hair et al., 2000).

\section{Resultados}

Los resultados se presentan en tres partes: una primera general y descriptiva de los datos en la que se evidencian tendencias generales, una segunda en la que construimos, mediante el análisis factorial, un agrupamiento y clasificación por tipos de funciones de los trabajadores sociales, y una tercera en la que, mediante el análisis de conglomerados, agrupamos a las trabajadoras sociales según el desempeño de las funciones en torno a cuatro perfiles profesionales mayoritarios. La validez de los grupos se demostró mediante un análisis de varianza (prueba de Levène y test de Tamhane).

\section{a) Tendencias generales}

En primer lugar, los resultados descriptivos apuntan tendencias generales, que luego concretamos cuando especificamos tipologías de trabajadoras sociales en torno a las funciones que desempeñan. Las principales tendencias generales que apuntan los datos descriptivos son tres:

a) Se constata la prevalencia de funciones de tipo asistencial y burocrático.

b) Se confirma el creciente desempeño de funciones orientadas a la gestión, planificación, coordinación y administración social.

c) Se evidencia la escasa implicación de las profesionales en la actividad investigadora.

El Trabajo Social es una actividad cuyos orígenes se encuentran en la atención al otro (en ayudar a mejorar la vida de las personas) y esta función sigue manteniéndose como un elemento definitorio y característico de la profesión, puesto que mayoritariamente $(84,4$ por ciento) la señalan como la principal función desempeñada. Sin embargo, los datos obtenidos en la valoración del resto de funciones nos hablan de las características administrativas y asistenciales de dicha atención (Gráfico 1).

Dos son los datos que apuntan a ello:

a) El 81,8 por ciento de los profesionales encuestados reconoce que su trabajo habitual se encuentra algo o bastante relacionado con la realización de tareas burocrático-administrativas que no están directamente relacionadas con el Trabajo Social.

b) El 63,7 por ciento de los profesionales reconoce que su trabajo diario tiene que ver algo o bastante con la función de coordinar servicios y medios.

Este hecho ya había sido indicado por numerosos profesionales del ámbito social con 


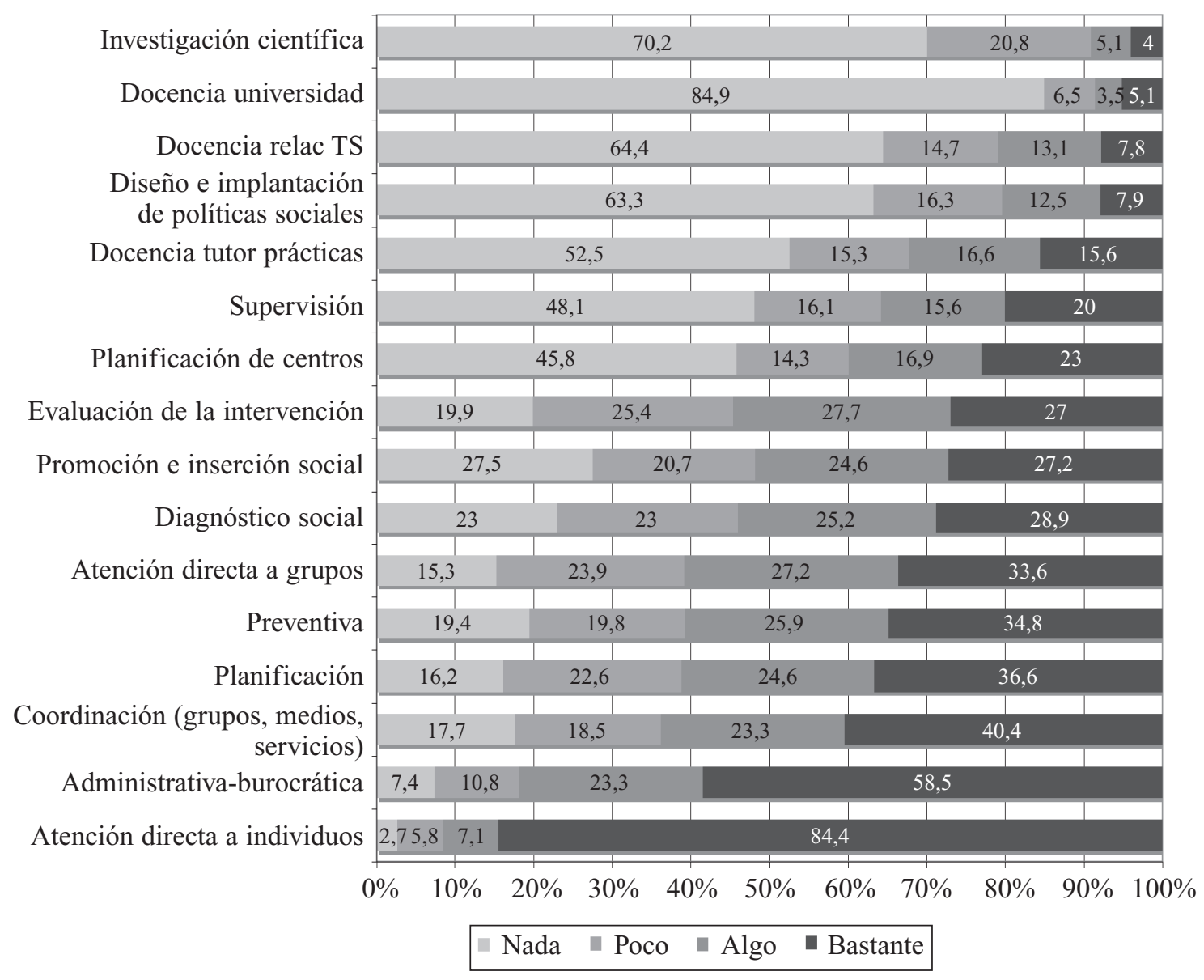

Gráfico 1. Clasificación de menor a mayor en grado de relevancia de las funciones desempeñadas por los trabajadores sociales en España.

Fuente: Grupo Efimec, proyecto FFI2008-005546, 2011.

anterioridad (Mendía, 1992; Hernández, 1992; Rossell y Pellegero, 1995) y también Sanz Citora (2001) señalaba que los servicios comunitarios especialmente se han convertido en gestores de una red de centros y en expendedores de servicios que, en la mayoría de los casos, ofrecen unos recursos escasos, siempre previa comprobación de medios y previa demostración de la necesidad. La obligación de comprobar la necesidad de los usuarios ha llevado a que las trabajadoras sociales dediquen gran parte de sus esfuerzos a tareas burocráticas y «a constantes procesos de control social» alejando su trabajo de los procesos educativos y de cambio más propios de la profesión del Trabajo Social. En la misma línea, Lima (2009), señala la necesidad de romper con la tendencia a la burocratización excesiva y a poner en valor las funciones de prevención, intervención global, mediación y a recuperar la dimensión comunitaria del Trabajo Social, para romper la cadena de «producto inmediato-cliente satisfecho» (Lima, 2009, p. 30). Esta actividad burocrática y administrativa, reglamentada por los poderes públicos, hace que la trabajadora social sea desplazada del proceso de hacer-paraconocer (reflexión), priorizando aspectos de inmediatez y de atención (operatividad). Así, funciones como el diagnóstico social, la evaluación o la investigación, son funciones que registran valores menos importantes de realización.

Por otra parte, y como señala Homs (2009), los datos nos muestran que la profesión se ha transformado en las últimas décadas, han aparecido con más fuerza funciones relacionadas con la gestión de equipamientos y servicios, donde tienen un peso mayor funciones como la 
planificación, la gestión, la planificación de centros, programas y servicios o la prevención.

La progresiva especialización de la intervención social ha dispersado la centralidad de un tipo de trabajadora social con una orientación de intervención comunitaria a diferentes tipos de trabajadoras sociales, que hacen frente a nuevos espacios laborales, a nuevas necesidades y a nuevos retos profesionales. A continuación, utilizamos el estudio multivariable de los datos obtenidos con el fin de identificar tipologías de funciones y tipos de perfiles profesionales de las trabajadoras sociales según realicen un determinado grupo de funciones.

\section{b) Análisis factorial: tipologías de funciones}

La praxis profesional es tan diversa y diversificada como los ámbitos en los que se desarrolla el Trabajo Social (Hernández Aristu, 2003). La mera enunciación y descripción de funciones, detallando los resultados obtenidos, nos informan de tendencias generales y confirman la complejidad de observar linealidades, patrones que permitan ordenar esa complejidad.

Para ello hemos realizado, en primer lugar, un análisis factorial de las variables, que nos ha servido para encontrar grupos homogéneos de las mismas a partir del conjunto total de funciones estudiado. La finalidad era reducir la dimensión de los datos y encontrar un número mínimo de dimensiones, que permitiera una mejor comprensión de los mismos. Así, del estudio factorial y utilizando el método de componentes principales como método de extracción (método de rotación, normalización Varimax), hemos reducido las dieciséis funciones observadas en torno a cuatro factores (Tabla 1).

Las variables incluidas en el primer factor muestran la existencia de funciones relacionadas con un contexto de la intervención profesional a nivel «macro»: planificación de centros, organización y control de programas o servicios, supervisión, diseño e implantación de políticas sociales para crear o reajustar servicios y coordinación de grupos medios y servicios.

El segundo factor observado agrupa variables en torno a funciones que se encuentran relacionadas con el contexto de la intervención a nivel «meso»: preventiva, planificación (diseño de proyectos y programas), atención directa a grupos, promoción e inserción social.
Las variables integradas en el tercer factor hacen referencia al contexto de «creación y difusión del conocimiento teórico y práctico del Trabajo Social»: docencia (profesora en universidad), docencia de cursos relacionados con el Trabajo Social, investigación científica, docencia como tutora de prácticas.

El cuarto factor agrupa solo tres variables que tienen que ver con funciones propias de la intervención profesional a nivel micro: atención directa a individuos, diagnóstico social y evaluación de la intervención.

\section{c) Análisis de conglomerados: tipología de perfiles profesionales según las funciones que desempeñan}

Una vez obtenida esta reducción dimensional, hemos procedido a guardar la posición de cada uno de los encuestados en torno a estos factores, reduciendo la complejidad de los datos iniciales. El segundo paso era obtener una clasificación por tipos, no ya de las variables, sino de las encuestadas en torno a diferentes grupos. Para ello hemos procedido a la realización de un estudio de conglomerados $\mathrm{K}$ medias (Cluster Analysis), técnica de análisis multivariante que agrupa los casos en función del parecido existente entre ellos, permitiendo identificar tipologías.

El estudio de K medias comienza con una división del conjunto de los datos en una serie de grupos « $\mathrm{X}$ » determinados al azar. Posteriormente se trata de mejorar esta primera clasificación reasignando aquellos elementos que tienen una mayor distancia al centroide del cluster al que pertenecen. El objetivo no es ahora explicar estadísticamente un determinado fenómeno, sino clasificar el conjunto de trabajadoras sociales muestreadas en un número reducido de grupos. Para ello hemos seleccionado los datos obtenidos mediante el análisis factorial ya comentado, tras un estudio pormenorizado de los mismos, habiendo reducido el número de variables y observando que la unidad de medida obtenida es la misma para todas las variables analizadas. Una vez llevado a cabo el proceso, se obtienen cuatro grupos característicos:

\section{Cluster 1: Perfil de gestión-planificación}

Este colectivo está formado por 181 profesionales (el 26 por ciento del total de la muestra). Es un colectivo cuyas funciones mayoritaria- 


\begin{tabular}{|l|c|c|c|c|}
\hline & Factor $\mathbf{1}$ & Factor $\mathbf{2}$ & Factor 3 & Factor 4 \\
\hline Supervisión & $\mathbf{0 , 7 8 4}$ & 0,077 & 0,156 & $-0,005$ \\
\hline $\begin{array}{l}\text { Planificación de centros, organización y control } \\
\text { de programas y/o servicios }\end{array}$ & $\mathbf{0 , 7 2 9}$ & 0,213 & 0,115 & 0,006 \\
\hline $\begin{array}{l}\text { Diseño e implantación de políticas sociales para crear } \\
\text { o reajustar servicios }\end{array}$ & $\mathbf{0 , 5 7 5}$ & 0,126 & 0,262 & 0,201 \\
\hline Coordinación (de grupos, medios, servicios, recursos) & $\mathbf{0 , 5 7 3}$ & 0,175 & 0,108 & 0,249 \\
\hline $\begin{array}{l}\text { Administrativa-burocrática no relacionada directamente } \\
\text { con la intervención profesional }\end{array}$ & $\mathbf{0 , 3 8 7}$ & 0,045 & $-0,296$ & 0,045 \\
\hline Preventiva & 0,114 & $\mathbf{0 , 7 5 1}$ & $-0,024$ & 0,339 \\
\hline Planificación & 0,365 & $\mathbf{0 , 7 1 1}$ & 0,112 & 0,121 \\
\hline Atención directa a grupos & $-0,07$ & $\mathbf{0 , 7 0 1}$ & 0,148 & $-0,038$ \\
\hline Promoción e inserción social & 0,191 & $\mathbf{0 , 3 9 6}$ & $-0,002$ & 0,315 \\
\hline Docente: profesor de Trabajo Social en universidad & 0,058 & 0,011 & $\mathbf{0 , 7 8 1}$ & $-0,004$ \\
\hline Docente: impartir cursos relacionados con el Trabajo Social & 0,100 & 0,161 & $\mathbf{0 , 7 2 6}$ & 0,089 \\
\hline $\begin{array}{l}\text { Investigación científica (crear conocimiento teórico } \\
\text { y metodológico) }\end{array}$ & 0,017 & 0,036 & $\mathbf{0 , 6 5 2}$ & 0,437 \\
\hline Docente: tutor de prácticas & 0,237 & 0,359 & $\mathbf{0 , 4 2 5}$ & $-0,232$ \\
\hline Diagnóstico social (investigación para la intervención) & 0,116 & 0,082 & 0,099 & $\mathbf{0 , 8 1 3}$ \\
\hline $\begin{array}{l}\text { Evaluación de la intervención constatando los resultados } \\
\text { obtenidos }\end{array}$ & 0,338 & 0,267 & 0,067 & $\mathbf{0 , 6 7 8}$ \\
\hline Atención directa a individuos & 0,154 & $-0,091$ & $-0,033$ & $\mathbf{0 , 5 1 3}$ \\
\hline
\end{tabular}

Tabla 1. Matriz de componentes rotados.

Fuente: Grupo Efimec, proyecto FFI2008-005546, 2011.

mente entroncan con lo que podríamos denominar administración social. Se trata de profesionales que dedican gran parte de su tiempo a labores logísticas y estratégicas de la acción social. Su trabajo se desarrolla más a un nivel macro y tiene que ver más con la práctica profesional indirecta que con la directa.

Este perfil se caracteriza por la importancia que presenta la realización de funciones tales como: coordinación de grupos, medios, servicios y recursos (98,9 por ciento), planificación (diseño de proyectos y programas) $(88,4$ por ciento), planificación de centros, organización y control de programas y servicios $(88,4$ por ciento), supervisión $(84,5$ por ciento) y evalua- ción de la intervención ( 80,1 por ciento). En todas ellas más del 80 por ciento de los individuos clasificados en este perfil dicen realizarlas en su trabajo diario (bastante o algo).

En cuanto a los rasgos socio-profesionales más significativos que definen este colectivo podemos destacar los siguientes:

- Es un perfil profesional mayoritariamente del ámbito de la gestión y dirección de los Servicios Sociales (el 60 por ciento de las que trabajan en este ámbito $(n=30)$ estarían dentro de este perfil), seguido de los servicios sociales (30 por ciento) y de los servicios sociales especializados $(27,2$ por ciento). 
- Es un perfil en el que la edad se convierte en una variable relevante, ya que conforme aumenta el rango de edad, aumenta la presencia de profesionales que encajan dentro de esta tipología: representa un 28 por ciento de los profesionales de entre 31 y 40 años, y un 31 por ciento de los profesionales entre 41 y 50 años.

- Al igual que la edad, los años de experiencia laboral también son importantes. Conforme aumentan los años de experiencia, aumenta igualmente la presencia de profesionales en torno a esta tipología; un 30 por ciento de las profesionales tienen entre 6 y 15 años de experiencia y un 30 por ciento tienen entre 16 y 25 años de experiencia.

- Se da una ligera mayor presencia masculina, un 35 por ciento de los varones encajan en este perfil por un 26 por ciento de las mujeres, confirmando a Grimwood, Popplestone y Phillipson (1993) cuando demostraron la sobrerrepresentación de los hombres en la gestión y la de las mujeres en las áreas prácticas.

\section{Cluster 2: Perfil de intervención para el cambio}

En términos numéricos, representa el tercer perfil en importancia, ya que agrupa al 24 por ciento de las encuestadas. Se caracteriza por agrupar a profesionales que desempeñan funciones relacionadas con la intervención directa para el cambio, atención directa a individuos (97,6 por ciento), diagnóstico social (investigación para la intervención) (93,9 por ciento) y evaluación de la intervención (79,4 por ciento). En este perfil las funciones de gestión decaen en importancia y ofrecen resultados más bajos, aunque las funciones burocráticas y administrativas, pese a no ser un rasgo esencial y definitorio de este perfil, registran un porcentaje de ejecución del 68,5 por ciento.

En cuanto a los rasgos socio-profesionales que caracterizan a las profesionales de este perfil podemos indicar que:

a) En el ámbito laboral este perfil se encuentra significativamente sobre-representado por profesionales que trabajan en el ámbito educativo (55 por ciento), en nuevos ámbitos profesionales (38 por ciento), en el ámbito del Tercer Sector ( 35 por ciento) y en menor medida en servicios sociales ( 27 por ciento) y en el ámbito de la salud (23 por ciento). El Libro
Blanco de Trabajo Social (Vázquez, 2004, p. 143) define como ámbitos nuevos de actuación los siguientes: planificación estratégica de servicios de bienestar, análisis y desarrollo organizacional, asesoramiento en la dirección de políticas de bienestar social, marketing social, comunicación e imagen en relación con temas sociales, atención a situaciones de catástrofes o de emergencias, defensa de los derechos humanos, cooperación y solidaridad internacional (proyectos de desarrollo), prevención de riesgos laborales, mediación familiar, terapia familiar, counseling, mediación comunitaria, peritaje social, y gestión y dirección de servicios y equipamientos sociales.

b) Son profesionales jóvenes. Encontramos un mayor porcentaje de profesionales con edades inferiores $y$, conforme aumenta la edad de las profesionales, disminuye su presencia en este perfil. Así encontramos un 35 por ciento de las profesionales de entre 20 y 30 años, por un 24 por ciento en el grupo de edad de los 31-40 años y un 18 por ciento en los grupos de más de 40 años.

c) Como era esperable, la experiencia laboral tiene que ver de forma directa con la edad, aunque no en igual medida, apreciándose mayor presencia de las profesionales más jóvenes.

\section{Cluster 3. Perfil de intervención asistencial}

Este tercer grupo es el mayoritario, agrupa al 41,1 por ciento de las profesionales encuestadas. Se caracteriza por registrar puntuaciones muy elevadas en funciones relacionadas con tareas de atención, gestión de servicios y prestaciones, que con tareas de intervención, de gestión o de docencia. Así, predominan la atención directa a individuos $(96,2$ por ciento) $\mathrm{y}$ las funciones administrativo-burocráticas (88,5 por ciento). Estas funciones serían las que mayoritariamente ocuparían la mayor parte de su tiempo, el resto de funciones registran valores inferiores al 55 por ciento. Destaca entre ellas una implicación clave en la tutoría de prácticas de estudiantes. El 30,9 por ciento de las encuestadas pertenecientes a este grupo destacan su implicación en esta tarea.

Respecto a los rasgos socio-profesionales que caracterizan los componentes de este perfil tenemos que señalar que:

a) Se encuentra una sobrerrepresentación en ámbitos laborales como justicia (67 por 
ciento), dependencia (58 por ciento), vivienda (43 por ciento) y servicios sociales especializados (44 por ciento). En todos ellos supera la media establecida del 41 por ciento.

b) Destaca que encontramos un menor número de varones ( 35 por ciento) que de mujeres (41 por ciento).

c) La experiencia laboral se convierte en una variable explicativa en tanto en cuanto indica que conforme aumenta la experiencia laboral disminuye la presencia de profesionales en este perfil.

\section{Cluster 4: Perfil académico}

Este agrupamiento presenta la representación poblacional más pequeña $(n=66)$ : el 9,4 por ciento de la muestra. Los factores que lo hacen característico, pese a no ser los mayoritarios, son los relacionados con una significativa mayor implicación en la docencia relacionada con el Trabajo Social y en la investigación que la observada respecto a la media. Así:

a) El 78,8 por ciento de las profesionales imparten cursos relacionados con el Trabajo Social (frente a un 7,6 por ciento de media para el conjunto de la muestra).

b) Un 74,2 por ciento imparte docencia en la Universidad, (frente al 18,7 por ciento de media del conjunto muestral).

c) Un 68,2 por ciento realizan tutorías de prácticas (frente al 31 por ciento de media del conjunto de la muestra).

d) Un 54,5 por ciento confirma que realiza tareas de investigación (frente a un 7,7 por ciento de media del conjunto de la muestra).

Al ser profesionales en activo, realizan de forma mayoritaria funciones que podrían estar incluidas en cualquiera de las tipologías anteriormente descritas. Así, observando los datos de la Tabla 2, podemos señalar que muchas de las profesionales que se clasifican dentro de este perfil tendrían mucho que ver con las funciones que discriminan al perfil de intervención para el cambio.

Rasgos socio-profesionales característicos de este perfil:

a) Destacamos la edad y la experiencia laboral como variables que explican la pertenencia a este perfil. A mayor edad, mayor porcentaje de profesionales que encontramos en este perfil: un 2 por ciento de las profesionales entre los 20 y los 30 años, frente al 23 por ciento de las profesionales con más de 50 años.

b) Igualmente ocurre con la experiencia laboral: un 6 por ciento de las profesionales con menos de cinco años de experiencia, frente al 21 por ciento de profesionales con más de 25 años de experiencia.

c) En cuanto a los ámbitos laborales, encontramos una mayor representación en el ámbito de la formación y el asesoramiento técnico (100 por ciento), en el sanitario (21 por ciento), en el de gestión y dirección de servicios sociales (20 por ciento), y en el Tercer Sector (19 por ciento) (Tabla 2).

\section{Discusión y conclusiones}

La práctica profesional del Trabajo Social es compleja. Su complejidad viene dada principalmente por la variedad de ámbitos y áreas de actuación profesional, la diversidad de actuaciones, ya sea a nivel individual, grupal o comunitario, y su diversidad teórico-metodológica. Estas complejidades se visualizan y tienen una concreción tangible en la diversidad de funciones que pueden desarrollar las profesionales del Trabajo Social en su labor cotidiana, concretándose estas de forma muy diversa en cada puesto profesional. Una trabajadora social debe planificar, evaluar, diagnosticar, investigar, coordinar servicios, coordinar recursos, administrar, asistir, mediar, etc. Una trabajadora social debe hacerlo todo. Es evidente que las profesionales en su desempeño diario no llevan a cabo todas las funciones que se les reconocen como propias de un desempeño profesional ideal, ajustándose a un perfil profesional único, sino que su actividad diaria se concreta en torno a una determinada serie de opciones. En la muestra estudiada hemos obtenido varias tendencias generales que nos hablan de la preeminencia de las funciones con orientación asistencial, administrativa y gestora. Funciones que pudieran dar la razón a aquellos (Healy, 2004) que hablan de una cierta desprofesionalización de las tareas realizadas por las trabajadoras sociales. O bien, confirmar lo ya expresado durante décadas anteriores en España por diferentes autores y profesionales del Trabajo Social, donde la burocratización y la tendencia al asistencialismo eran consecuencia de una determinada organización de los servicios so- 


\begin{tabular}{|c|c|c|c|c|c|c|}
\hline & $\mathbf{N}$ & $\begin{array}{c}\text { Tipo 1 } \\
\text { Gestión- } \\
\text { planificación } \\
\text { n (\%) }\end{array}$ & $\begin{array}{c}\text { Tipo 2 } \\
\text { Intervención } \\
\text { para el cambio } \\
\text { n (\%) }\end{array}$ & $\begin{array}{c}\text { Tipo } 3 \\
\text { Intervención } \\
\text { asistencial } \\
\text { n (\%) }\end{array}$ & $\begin{array}{c}\text { Tipo } 4 \\
\text { Académico } \\
\text { n (\%) }\end{array}$ & $\begin{array}{l}\text { Total } \\
(\%)\end{array}$ \\
\hline \multicolumn{7}{|l|}{ Sexo } \\
\hline Hombre & 63 & $16(35 \%)$ & $15(20 \%)$ & $26(35 \%)$ & $6(10 \%)$ & $100 \%$ \\
\hline Mujer & 637 & $165(26 \%)$ & $150(24 \%)$ & $262(41 \%)$ & $60(9 \%)$ & $100 \%$ \\
\hline \multicolumn{7}{|l|}{ Edad } \\
\hline Entre 20 y 30 años & 124 & $26(21 \%)$ & $43(35 \%)$ & $53(43 \%)$ & $2(2 \%)$ & $100 \%$ \\
\hline Entre 31 y 40 años & 277 & $78(28 \%)$ & $67(24 \%)$ & $112(40 \%)$ & $20(7 \%)$ & $100 \%$ \\
\hline Entre 41 y 50 años & 207 & $64(31 \%)$ & $38(18 \%)$ & $82(40 \%)$ & $23(11 \%)$ & $100 \%$ \\
\hline Más de 50 años & 92 & $13(14 \%)$ & $17(18 \%)$ & $41(45 \%)$ & $21(23 \%)$ & $100 \%$ \\
\hline \multicolumn{7}{|l|}{ Ámbito de intervención } \\
\hline Servicios Sociales & 204 & $61(30 \%)$ & $55(27 \%)$ & $83(41 \%)$ & $5(2 \%)$ & $100 \%$ \\
\hline Servicios Sociales especializados & 239 & $65(27 \%)$ & $47(20 \%)$ & $106(44 \%)$ & $21(9 \%)$ & $100 \%$ \\
\hline Salud & 103 & $11(11 \%)$ & $24(23 \%)$ & $46(45 \%)$ & $22(21 \%)$ & $100 \%$ \\
\hline Educación & 20 & $4(20 \%)$ & $11(55 \%)$ & $5(15 \%)$ & - & - \\
\hline Justicia & 6 & 0 & $1(17 \%)$ & $4(67 \%)$ & $1(17 \%)$ & $100 \%$ \\
\hline Vivienda & 14 & $4(29 \%)$ & $4(25 \%)$ & $6(43 \%)$ & - & - \\
\hline Tercer Sector & 26 & $6(23 \%)$ & $9(35 \%)$ & $6(23 \%)$ & $5(19 \%)$ & $100 \%$ \\
\hline Formación y Asesoría Técnica & 5 & - & - & - & $5(100 \%)$ & $100 \%$ \\
\hline Nuevos ámbitos & 8 & $4(50 \%)$ & $3(38 \%)$ & 0 & $1(13 \%)$ & $100 \%$ \\
\hline $\begin{array}{l}\text { Gestión y dirección de Servicios } \\
\text { Sociales }\end{array}$ & 30 & $18(60 \%)$ & - & $6(20 \%)$ & $6(20 \%)$ & $100 \%$ \\
\hline Dependencia & 45 & $8(18 \%)$ & $11(24 \%)$ & $26(58 \%)$ & - & - \\
\hline \multicolumn{7}{|l|}{ Años de experiencia } \\
\hline Menos de 5 años de experiencia & 175 & $33(19 \%)$ & $54(31 \%)$ & $77(44 \%)$ & $11(6 \%)$ & $100 \%$ \\
\hline Entre 6 y 15 años de experiencia & 263 & $78(30 \%)$ & $62(24 \%)$ & $110(42 \%)$ & $13(5 \%)$ & $100 \%$ \\
\hline Entre 16 y 25 años de experiencia & 199 & $59(30 \%)$ & $34(17 \%)$ & $77(39 \%)$ & $29(15 \%)$ & $100 \%$ \\
\hline Más de 25 años de experiencia & 63 & $11(17 \%)$ & $15(24 \%)$ & $24(38 \%)$ & $13(21 \%)$ & $100 \%$ \\
\hline Totales perfiles & & $185(26 \%)$ & $165(24 \%)$ & $288(41 \%)$ & $66(9 \%)$ & $\begin{array}{c}700 \\
(100 \%)\end{array}$ \\
\hline
\end{tabular}

Tabla 2. Funciones realizadas según los diferentes perfiles profesionales obtenidos. Rasgos socio-profesionales según perfil.

Fuente: Grupo Efimec, proyecto FFI2008-005546, 2011. 
ciales: «los trabajadores sociales trabajan más con papeles que con personas» (Estruch y Guell, 1976). Este hecho no es exclusivo de nuestro país también se puede constatar en otro países. Concretamente Stataham (2009, p. 3) se refiere al informe, Social Work Task Force, 2009, para señalar que las trabajadoras sociales no tienen tiempo suficiente: «to devote directly to the people they want to help, and feel "tied up in bureacracy"».

Los datos también nos hablan de la existencia de un perfil con funciones centradas en la gestión y planificación de los servicios sociales. Estas funciones han crecido en importancia y relevancia durante los últimos años, debido en gran parte a los cambios normativos y al crecimiento del sector privado tanto lucrativo como no lucrativo. La tendencia actual hacia una gestión descentralizada e indirecta en la provisión de los servicios sociales ha generado que las actividades a las que corresponden las funciones de planificación, elaboración de presupuestos, coordinación y control de equipos y empleados, la diferenciación y distribución de roles y funciones dentro de la propia organización, así como a la evaluación interna y/o externa de la eficacia y eficiencia de los programas, de los proyectos, etc. hayan tomado mayor importancia en las actividades profesionales del Trabajo Social.

Por otro lado, aquellos perfiles con funciones más reflexivas y de contacto directo con la profesión, como la intervención para el cambio (agentes de cambio) y la reflexión sobre la acción (investigación), son las que obtienen menor grado de representatividad en la muestra estudiada.

Del estudio de las funciones profesionales, y en el marco teórico de la teoría de la acción comunicativa de Habermas, parece inferirse que se cumple el hecho de que el Trabajo So- cial se ha convertido en un subsistema dirigido a evitar o superar disfunciones sistémicas (falta de dinero, de vivienda, de atención y de otros recursos), donde cobran especial relevancia elementos propios de las lógicas sistémicas (Habermas, 1987) como la gestión, la administración y la burocracia (indirect social work activities). Mientras que las funciones más centradas en el sujeto, the primary activity"face to face» (Broadhurst et al., 2010), propias del mundo de la vida y enraizadas en los orígenes del Trabajo Social como el entendimiento, la participación, la emancipación y la comunicación, parece que perderían relevancia sobre las lógicas sistémicas. Se priorizan las necesidades del sistema (acciones racionales administrativa y económicamente) sobre la atención a las necesidades del sujeto (comprensión comunicativamente mediada). Todos estos datos plantean varias líneas de reflexión y de discusión. La primera de ellas apunta a la reflexión sobre el futuro de la actividad de las trabajadoras sociales en la sociedad, analizando primero el encargo social y después la identidad profesional, delimitando bien las funciones con las de otras profesionales. La segunda línea reflexiva y de discusión tiene que ver con la orientación académica del Grado en Trabajo Social, ya que la formación universitaria puede atender a las necesidades que impone el «mercado laboral» de lo social en cada momento, en el que parecen imponerse determinadas funciones con marcado carácter administrativo y de gestión o puede formar para el conjunto de funciones que la tradición reflexiva de la disciplina (investigación) y la evolución histórica de la profesión han ido configurando como esenciales de la misma (intervención). Queda aquí la invitación a la profesión para reflexionar sobre estos aspectos, una vez estudiados los datos.

\section{Referencias bibliográficas}

Boody, J. y Statham, J. (2009). European Perspectives on Social Work: Models of Education and Professional Roles. A Briefing Paper. Londres: Thomas Coram Research Unit.

Broadhurst, K. et al.(2010). Performing 'Initial Assessment': identifying the latent conditions for error at the front door of local authority children's services. British Journal of Social Work, 40 (2), 352-370.

Estruch, J. y Güell, A. (1976). Sociología de una profesión. Los asistentes sociales. Barcelona: Ediciones Península.

Gómez, R. (2010). Situación del Trabajo Social en Castilla y León. Cuadernos de Trabajo Social, 23, 211-227. 
Grimwood, C.; Popplestone, R. y Phillipson, J. (1993). Women, Management and Care. Basingstoke (Hampshire): Palgrave MacMillan.

Habermas, J. (1987). Teoría de la acción comunicativa. Madrid: Taurus.

Hair, J., Anderson, R., Tatham, R. y Black, W. (2000). Análisis Multivariante. Madrid: Prentice Hall.

Harlow, E. (2000). Introduction: Postmodernisation and Change in Social Work and Social Welfare. En E. Harlow y L. Lawler (eds.), Management, Social Work and Change (pp. 5-32). United Kingdom: Ashgate.

Healy, K. y Meagher, G. (2004). The Reprofessionalization of Social Work: Collaborative Approaches for Achieving Professional Recognition. British Journal of Social Work, 34 (2), 243-260.

Hernández Aristu, J. (1991). Acción comunicativa e intervención social, Madrid: Editorial Popular.

Hernández Aristu, J. (2004). Trabajo Social en la Postmodernidad, Zaragoza: Certeza.

Holmes, L., McDermid, S., Jones, A. y Ward, H. (2009). How social workers spend their time. (Research Report DCSF-RR087). Nottingham: Loughborough University, Department for Children, Schools and Families.

Homs, O. (2009).Una nueva generación de trabajadores sociales y un nuevo modelo de servicios sociales. Revista de Servicios Sociales y Política Social, 86, 11-113.

Kornbeck, J. (2005). The Bologna Process in Europe: a challenge for social work training in Germany. Social Work Education, 24, 716-718.

La Valle, I. y Lyons, K. (1996). The Social Worker speaks: the perceptions of recent changes in British social work. Practice, 8 (2), 5-14.

Lawler, J. (2000). The rise of Managerialism in Social Work. En E. Harlow y L. Lawler (eds.) Management, Social Work and Change (pp. 33-57). United Kingdom: Ashgate.

Lima, A. (2009). La profesión del Trabajo Social. Revista de Servicios Sociales y Política Social, $86,9-42$.

Lorenz, W. (2005). Social Work and the Bologna Process. Social Work and Society, 3 (2), 224-235.

Mendía Gallardo, R. (1992). Un modelo focalizado de formación permanente de trabajadores de servicios sociales. Zerbituan: Gizarte zerbitzuetarako aldizkaria, 18-19, 4-12.

Parton, N. y O’Byrne, P. (2000). Constructive Social Work: Towards a New Practice, Basingstoke (Hampshire): MacMillan Press.

Portuondo, F. (1983). Economía de las empresas industriales. La Habana: Editorial Pueblo y Educación.

Rossell, T. y Pellegero, N. (1995). Modelos, métodos, técnicas y habilidades para la atención directa en Trabajo Social. Revista de Servicios Sociales y Política Social, 20, 98-109.

Sanz Cintora, A. (2001). Acción Social y Trabajo Social en España. Una revisión histórica, Acciones e Investigaciones Sociales, 13, 5-42.

Social Work Task Force. (2009). Facing up to the task. (The interim report of the Social Work Task Force). England: Secretary of State for Health and the Secretary of State for Children, Schools and Families. Recuperado de http://www.swap.ac.uk/docs/taskforceinterim_rpt.pdf

Sonnenberg, K. (2003). The Social Work Profession as Qualified by the Aspects of Efficiency and Ethics: A Comparison of Germany and England. Birmingham: Venture Press.

Stataham, J. y Boddy, J. (2009). Social Work: models of educational and professional roles. Nuffield Foundation. (Briefing Paper).

Torres Esperón, M. (2008). Metodología para definir funciones profesionales». Revista Cubana de Salud Pública, 34 (4). Recuperado de http://tinyurl.com/3kyabtr

Úriz, M.J., Ballestero, A. y Urien, B. (2007). Dilemas éticos en la intervención social. Zaragoza: Mira Editores.

Vázquez, O. (Coord.) (2004). Título de Grado en Trabajo Social (Libro blanco). Madrid: Aneca. 